\title{
Bio-based production of crotonic acid by pyrolysis of poly(3-hydroxybutyrate) inclusions
}

\begin{abstract}
Bio-based material development has become a new focus globally due to limited supply, increasing price of fossil fuel, and demands for environment sustainability. Current industrial production of crotonic acid through petrochemical route has several drawbacks: i) nonrenewable, as it is derived from petroleum resource, ii) involves numerous complicated steps, and iii) produces low yield. Therefore, this paper proposes a method for production of biobased crotonic acid by direct pyrolysis of bacterial poly(3-hydroxybutyrate) inclusion as an alternative to the petrochemical route. Thermogravimetric profile of poly(3-hydroxybutyrate) inclusions showed poly(3-hydroxybutyrate) degradation occurred at a temperature range of $270{ }^{\circ} \mathrm{C}-350{ }^{\circ} \mathrm{C}$ with maximum degradation rate at $310{ }^{\circ} \mathrm{C}$. Analysis of products from isothermal pyrolysis of poly(3-hydroxybutyrate) at $310{ }^{\circ} \mathrm{C}$ revealed that pyrolysis of poly(3hydroxybutyrate) inclusions yielded approximately $63 \%$ of crotonic acid. This is $30 \%$ higher than the conventional crotonic acid production via petrochemical method. The proposed method also offers other benefits such as renewable and simpler in processing. Besides, byproducts of fermentation and pyrolysis are easy to treat, thus minimizing threat to the environment. Moreover, demands for bio-based products are expected to rise in the near future because of social, environmental and economical issues related to fossil resources which make bio-based production method more appealing and favourable. Therefore, pyrolysis of bacterial poly(3-hydroxybutyrate) inclusions provides new insight of renewable and green chemistry of the crotonic acid production.
\end{abstract}

Keyword: Crotonic acid; Poly(3-hydroxybutyrate); Pyrolysis; Bio-based product 